very or quite worried about abduction or molestation, and $89 \%$ were very or quite worried about traffic. The strongest predictors of car travel to school were car ownership, greater distance to school, attendance at an independent school, and parental worry about abduction (table). For the journey home, the strongest predictors were greater distance to school, car ownership, and attending an independent school.

\section{Comment}

Distance to school and car ownership were principal determinants of car travel. After adjustment for these factors, children at independent schools were still more likely to travel by car. Parental fear about "stranger danger" also influenced the decision to drive children to school.

Although few translated questionnaires were requested, the study population adequately represented the ethnic distribution of children attending school in the two boroughs. Our results might appropriately be generalisable to other urban primary school populations.

Increasing emphasis on school choice has been accompanied by a $20 \%$ increase in average distance travelled to school. ${ }^{1}$ Policies that encourage children to attend nearby schools are likely to reduce car travel and increase walking. Parents who currently drive their children might forgo the car for safe, convenient alternatives that address their fears. Unless such alternatives are developed, parents who do not currently drive to school are likely to do so when the option becomes available.

This study originated from joint work with the Camden and Islington Accident Prevention Alliances.

Contributors: $\mathrm{CD}$ designed the protocol and questionnaire, implemented the study, and participated in the study design, data analysis and interpretation, and writing the paper. IR initiated the research and participated in study and questionnaire design, data analysis and interpretation, and writing the paper. LL performed data analysis and edited the paper. DA managed and audited the data, performed preliminary analyses, and edited the paper. We received helpful advice on questionnaire and study development from Mayer Hillman, Belita Clahar, Michelle Walsh, and Suzanne Slater, and on statistical analysis from David Dunn. We acknowledge the assistance of Jackie Payne, Elaine Morrison, the local education authorities in Camden and Islington, and the participating schools, teachers, and pupils.

Funding: The London Boroughs of Camden and Islington funded the study. The Camden and Islington Health Authority funded CD and DA.

Conflict of interest: None.

1 Department of Transport. Transport statistics report: national travel survey 1992/94. London: HMSO, 1995.

2 British Medical Association. Road transport and health. London: BMA, 1997.

3 Hillman M, ed. Children, transport and the quality of life. London: PSI Publishing, 1993.

4 Roberts I, Carlin J, Bennett C, Bergstrom E, Guyer B, Nolan T, et al. An international study of the exposure of children to traffic. Injury Prev 1997;3:89-93.

5 Hillman M, Adams J, Whitelegg J. One false move. . . a study of children's independent mobility. London: PSI Publishing, 1990.

(Accepted 5 February 1998)

\title{
Evaluation of indices of obesity in men: descriptive study
}

\author{
Derrick Pounder, David Carson, Michael Davison, Yoshiyuki Orihara
}

Department of Forensic Medicine, Dundee Royal Infirmary, Dundee DD1 9ND

Derrick Pounder, professor

David Carson, research student Michael Davison, research student Yoshiyuki Orihara research fellow

Correspondence to: Professor Pounder

BMJ 1998;316:1428-9
The intra-abdominal accumulation of fat is more common in men; it is an independent risk factor for diabetes mellitus and cardiovascular disease. ${ }^{1}$ The assessment of obesity usually focuses on abdominal fatness, but the most appropriate clinical measurement of obesity has been disputed..$^{2}$ We evaluated several measures of obesity by comparing them with observed intra-abdominal and subcutaneous fat during postmortem examination.

\section{Methods and results}

A series of 100 men was studied. Cases of prolonged hospitalisation, severe trauma, chronic wasting disease, and postmortem decomposition were excluded from analysis. Measurements were made using techniques suitable for supine cadavers. Body weight was measured to within $1 \mathrm{~kg}$, body length to within $1 \mathrm{~cm}$, hip circumference at the iliac crests to within $1 \mathrm{~cm}$, and waist circumference at the umbilicus to within $1 \mathrm{~cm}$. Uniform cores of subcutaneous fat were obtained from over the biceps, anterior thigh, anterior chest, and anterior abdomen. These samples were weighed to within $0.1 \mathrm{~g}$. The greater omentum was excised as was the pararenal fat; these were weighed to within $1 \mathrm{~g}$.
The mean age at the time of death was 52.8 years (median 55.5, range 17 to 89 , interquartile range 37 to 67). Mean body weight was $74 \mathrm{~kg}$ (median 73, range 39 to 142 , interquartile range 65 to 83 ). In nine cases the body mass index (weight $(\mathrm{kg}) /$ height $\left(\mathrm{m}^{2}\right)$ ) was $<20$; in 50 it was 20 to 24 ; in 32 it was 25 to 29 ; in 8 it was 30 to 39 ; and in 1 it was $\geqslant 40$. Waist circumference was $<94 \mathrm{~cm}$ in 69 cases; 94 to $101 \mathrm{~cm}$ in 19 , and $\geqslant 102 \mathrm{~cm}$ in 12.

The strongest correlation between the weight of the subcutaneous fat taken from the four sites was between the fat at the abdomen and that at the chest (0.60); the weakest correlation was between the fat at the abdomen and that at the upper arm (0.36). The correlation between the weight of the intra-abdominal fat and the weight of the subcutaneous fat was 0.57 for the anterior chest, 0.38 for the abdomen, 0.45 for the upper arm, and 0.28 for the thigh. When 82 cases with body mass index between 20 and 30 were analysed, the correlation was 0.49 for the anterior chest, 0.23 for the abdomen, 0.39 for the upper arm, and 0.15 for the thigh.

When the weight of intra-abdominal fat was used as the outcome $r^{2}$ predictive value was $40 \%$ for body weight, $37 \%$ for body mass index, $61 \%$ for waist circumference, $47 \%$ for hip circumference, $43 \%$ for 
waist to hip ratio, and $54 \%$ for waist to height ratio. For the 82 cases with body mass index between 20 and 30 the $r^{2}$ predictive value was 32\% for body weight, $31 \%$ for body mass index, $58 \%$ for waist circumference, $40 \%$ for hip circumference, $38 \%$ for waist to hip ratio, and $51 \%$ for waist to height ratio. Waist circumference was strongly correlated with body weight $(r=0.90)$, body mass index $(r=0.90)$, and hip circumference $(r=0.95)$. For the 82 cases with body mass index between 20 and $30 r$ was 0.81 for body weight, 0.80 for body mass index, and 0.91 for hip circumference. When non-obese cases were grouped by waist circumference or body mass index a trend of increasing intraabdominal fat mass was seen (table).

\section{Comment}

Body mass index is the traditional measure of obesity; it is an index of weight relative to stature. The waist to hip circumference was the first index adopted as a measure of the abdominal location of body fat. ${ }^{1}$ The results of studies that used computed tomography have suggested that waist circumference correlates better with abdominal visceral fat mass. ${ }^{2}$ Advocates of using waist circumference to measure obesity argue that it is a straightforward measurement that relates to both body weight and the distribution of fat. ${ }^{3}$

Indices of obesity can be evaluated during postmortem examination by comparing them with the amount of body fat observed. Measurements cannot be made in the same manner as during clinical practice, so values observed at necropsy may not correspond exactly with clinical indices. Within these limitations this study confirms that waist circumference correlates more strongly with intra-abdominal fatness than any other suggested index, and that this is true across the range of body mass index values.

When the cases were grouped together (table) a significant increase in intra-abdominal fat mass occurred before the point at which guidelines in the United Kingdom recommend implementation of a weight loss programme-that is, waist measurement of $94 \mathrm{~cm}$ and body mass index $>25$. The appropriateness of using a
Mean, median, and range of weight $(\mathrm{g})$ of intra-abdominal fat from the greater omentum and pararenal area in 59 non-obese subjects by body mass index $\left(\mathrm{kg} / \mathrm{m}^{2}\right)$ and waist circumference $(\mathrm{cm})$ at the umbilicus

\begin{tabular}{lrrrr} 
& $\begin{array}{l}\text { No of } \\
\text { cases }\end{array}$ & Mean & Median & Range \\
\cline { 5 - 5 } & & & & \\
\hline Body mass index $\left(\mathrm{kg} / \mathrm{m}^{2}\right):$ & 7 & 629 & 655 & 207 to 1318 \\
\hline 20 & 9 & 661 & 736 & 298 to 1128 \\
\hline 21 & 11 & 957 & 691 & 186 to 2220 \\
\hline 22 & 10 & 1338 & 1159 & 331 to 2633 \\
\hline 23 & 13 & 1432 & 1355 & 511 to 3765 \\
\hline 24 & 9 & 1656 & 1762 & 622 to 3626 \\
\hline 25 & & & & 207 to 1246 \\
\hline Waist circumference $(\mathrm{cm}):$ & 10 & 556 & 484 & 402 to 1759 \\
\hline $74-77$ & 14 & 865 & 761 & 331 to 2170 \\
\hline $78-81$ & 13 & 994 & 876 & 511 to 2220 \\
\hline $82-85$ & 13 & 1243 & 1250 & 1113 to 3626 \\
\hline $86-89$ & 9 & 2057 & 1963 &
\end{tabular}

body mass index of 25 to determine the point at which weight reduction should occur has been questioned, ${ }^{4}$ and it has also been suggested that the ideal body mass index should be about 22. ${ }^{5}$ Above 22 some men accumulate large amounts of intra-abdominal fat and the health risks associated with this fat.

Contributors: DP initiated the study, designed the protocol, helped analyse data, wrote the paper, and is guarantor for the study. MD helped design the protocol, review the literature, collect and analyse the data. DC and YO collected and analysed data.

Funding: The Crown Office funds the department of forensic medicine.

Conflict of interest: None

1 Bjorntorp P. Abdominal fat distribution and disease: an overview of epidemiological data Ann Med 1999:24:15-8.

2 Pouliot M-C, Despres J-P, Lemieux S, Moorjani S, Bouchard C, Tremblay A, et al. Waist circumference and abdominal sagittal diameter: best simple anthropometric indices of abdominal visceral adipose tissue accumulation and related cardiovascular risk in men and women. Am J Cardio 1994;73:460-8.

3 Lean MEJ, Han TS, Morrison CE. Waist circumference as a measure for indicating need for weight management. $B M J$ 1995;311:158-61.

4 Tunstall-Pedoe H. Contour control, survival, and quality of life. BMJ 1997;314:1291-2.

5 Shaper AG, Wannamethee SG, Walker M. Body weight: implications for the prevention of coronary heart disease, stroke, and diabetes mellitus in a cohort study of middle aged men. BMJ 1997;314:1311-7.

(Accepted 31 October 1997)

\section{Fifty years ago}

\section{The new NHS: National Health Service. Mr. Bevan's statement}

The Minister of Health made the following statement in the House of Commons on Wednesday, April 7:

I think that the time has come for me to make a statement to the House about the medical profession and the National Health Service. Needless to say, I make this statement on behalf both of the Secretary of State for Scotland and myself, and of the Government as a whole.

I have been receiving representations from many quarters, and I have studied the resolutions which both the Royal College of Physicians and the British Medical Association have sent on to me-and which have since been published and are familiar to the House. Meanwhile I have been trying, so far as I am able, also to determine for myself what it is that is really and sincerely worrying the doctor.

No doubt, as we gain experience, we shall find many modifications which would improve the scheme. That is always true of major legislation of this kind. But it seems to me that it is somewhere beyond all this that the key to the doctors' unease and restlessness lies, and that it consists of some instinctive fear-shared by many most well-meaning men and women- that, although the Act does not propose it and although the Government have themselves denied it, the real objective is a full-time, salaried State medical service. It is this fundamental point which I want now to tackle once and for all. As long ago as 1946, in this House, I said:

"Some doctors have expressed the fear that this is merely the beginning of a full-time salaried service. I cannot read into the mind of any future Minister or prophesy what may be done by future Governments, but that is not our intention." (Mr Bevan's statement, 17 April 1948, p 763. See also editorial by Gordon Macpherson, 3 January 1998, p 6.) 